\title{
Actividad de los extractos acuosos liofilizados de hojas y corteza de Jodina rhombifolia (Hook. \& Arn.) Reissek (Santalaceae) sobre el tránsito intestinal en ratones
}

\author{
Mauricio Roberto Teves*, Farm., Graciela Haydée Wendel, Ph.D. \\ ${ }_{1}$ Farmacología, Departamento de Farmacia, Facultad de Química, Bioquímica y Farmacia, \\ Universidad Nacional de San Luis, Argentina
}

\begin{abstract}
Recibido: 23 de junio del $2016 \quad$ Aprobado: 10 de noviembre del 2017
*Autor de correspondencia: Mauricio Roberto Teves. Facultad de Química, Bioquímica y Farmacia, Chacabuco y Pedernera, Universidad Nacional de San Luis, D5700BWS, San Luis, Argentina. Tel: +54 2664520300 interno 6130. Correo electrónico: maurote@unsl.edu.ar
\end{abstract}

Cómo citar este artículo: Teves MR. Actividad de los extractos acuosos liofilizados de hojas y corteza de Jodina rhombifolia (Hook. \& Arn.) Reissek (Santalaceae) sobre el tránsito intestinal en ratones. Spei Domus. 2016;12(25):1-8.

Resumen. Introducción: Jodina rhombifolia (Hook. \& Arn.) Reissek (Santalaceae) es utilizada en la terapéutica popular para el tratamiento de una gran diversidad de problemas de salud que afectan al metabolismo y al sistema digestivo, respiratorio, músculo-esquelético, cardiovascular y genitourinario. Nuestro objetivo es aportar datos que permitan contribuir a la validación de su uso como antidiarreico. Metodología: conforme a referencias bibliográficas, utilizamos el extracto acuoso de la corteza, el extracto acuoso de las hojas y el empleo conjunto de ambos extractos para ensayar los efectos resultantes sobre la actividad intestinal de ratones Rockland. Resultados: la administración de los extractos de hojas y de corteza por separado no arrojaron valores significativos; sin embargo, la administración conjunta de ambos extractos a las dosis de 125, 250 y $500 \mathrm{mg} / \mathrm{kg}$ resultaron en una disminución significativa - y de manera dependiente de la dosis - del tránsito intestinal en ratones Rockland $\mathrm{p}<0,05 ; \mathrm{p}<0,01 \mathrm{y} \mathrm{p}<0,01$, respectivamente, en comparación con el grupo de control negativo. Conclusiones: los resultados obtenidos nos demuestran que la utilización conjunta del extracto acuoso de las hojas y de la corteza de J. rhombifolia poseen un significante efecto reductor del tránsito intestinal normal en el modelo in vivo utilizado y que avala la utilización en la medicina popular argentina.

Palabras clave: extracto acuoso de corteza, extracto acuoso de hojas, Jodina rhombifolia, tránsito intestinal. 


\title{
Activity of Lyophilized Aqueous Extracts of Jodina rhombifolia (Hook. \& Arn.) Reissek (Santalaceae) Leaves and Bark on Intestinal Transit In Mice
}

\begin{abstract}
Introduction: Jodina rhombifolia (Hook. \& Arn.) Reissek (Santalaceae) is a part of popular therapy for treating a wide range of health problems that affect metabolism and the digestive, respiratory, musculoskeletal, cardiovascular and genitourinary systems. Our aim is to provide data that contribute to validating its use as an antidiarrheal. Materials and methods: According to the literature, we used the aqueous extract of bark, the aqueous extract of leaves and a combination of both to test the resulting effects on the intestinal activity of Rockland mice. Results: The administration of leaf and bark extracts separately did not yield significant values; however, co-administration of both extracts at doses of 125 , 250 and $500 \mathrm{mg} / \mathrm{kg}$ resulted in a significant decrease - and in a dose-dependent mannerof intestinal transit in Rockland mice ( $<<0.05 ; \mathrm{p}<0.01$ and $\mathrm{p}<0.01$, respectively), compared to the negative control group. Conclusions: The results obtained show us that the joint use of aqueous extract of $J$. Rhombifolia leaves and bark has a reducing effect on normal intestinal transit in the in vivo model and supports its use in Argentine popular medicine.
\end{abstract}

Keywords: aqueous extract of bark, aqueous extract of leaves, Jodina rhombifolia, intestinal transit.

\section{Atividade dos extratos aquosos liofilizados de folhas e casca de Jodina rhombifolia (Hook. \& Arn.) Reissek (Santalaceae) sobre o trânsito intestinal em ratos}

Resumo. Introdução: Jodina rhombifolia (Hook. \& Arn.) Reissek (Santalaceae) é utilizada na terapêutica popular para o tratamento de problemas de saúde que afetam o metabolismo e o sistema digestivo, respiratório, músculo-esquelético, cardiovascular e genitourinário. Nosso objetivo é fornecer dados que permitam contribuir para a validação de seu uso como antidiarreico. Metodologia: conforme referências bibliográficas, utilizamos o extrato aquoso da casca, o extrato aquoso das folhas e o emprego conjunto de ambos os extratos para ensaiar os efeitos resultantes sobre a atividade intestinal de ratos Rockland. Resultados: a administração dos extratos de folhas e de casca por separado não demonstram valores significativos; contudo, a administração conjunta de ambos às doses de 125, 250 e $500 \mathrm{mg} / \mathrm{kg}$ resultaram numa diminuição significativa —e de maneira dependente da dose- do trânsito intestinal em ratos Rockland $\mathrm{p}<0,05 ; \mathrm{p}<0,01$ e $\mathrm{p}<0,01$, respectivamente, em comparação com o grupo de controle negativo. Conclusões: os resultados obtidos nos demonstram que a utilização conjunta do extrato aquoso das folhas e casca de J. rhombifolia possuem significante efeito redutor do trânsito intestinal normal no modelo in vivo utilizado e que garante a utilização na medicina popular argentina.

Palavras-chave: extrato aquoso de casca, extrato aquoso de folhas, Jodina rhombifolia, trânsito intestinal. 


\section{Introducción}

La utilización de variadas y cuantiosas especies vegetales con propósitos curativos resulta ser una práctica empírica que durante centenares de años proveyó a los habitantes de nuestro mundo de una fuente valiosa de principios activos naturales, siendo el principal e incluso, en ciertas oportunidades, único medio de subsistencia para combatir situaciones referidas a cuestiones de salud de la vida cotidiana. Actualmente, los remedios naturales representan un recurso que en considerables situaciones poblacionales y sociales es imprescindible, motivado por la incapacidad económica de acceder a formas farmacéuticas medicamentosas desarrolladas y presentadas para su expendio.

Es importante la actuación que la medicina tradicional representa en la asistencia sanitaria, principalmente en la atención primaria de la salud; por otro lado, debe ser tenido en cuenta que su utilización constituye un procedimiento de elevada frecuencia en algunos países y de creciente costumbre en otros, tal como lo declara la Organización Mundial de la Salud [1].

En la medicina tradicional suramericana, la especie vegetal Jodina rhombifolia (Hook. \& Arn.) Reissek (Santalaceae), conocida comúnmente en Argentina con los nombres vulgares "peje", "quebrachillo", "quebracho flojo" y "sombra de toro" [2-5], goza de gran popularidad en las regiones donde se desarrolla para el tratamiento de una gran diversidad de problemas de salud que afectan al metabolismo y al sistema digestivo, respiratorio, músculo-esquelético, cardiovascular y genitourinario [5-26]. En lo que se refiere a su práctica popular en la terapia digestiva, son vastos los registros bibliográficos que denotan el uso de la corteza para el tratamiento de la disentería [5], [8], [14], [15], [25], [27-29] y de hojas y corteza [7], [22]. A su vez, Hurrell et al. [14] reportan el uso de la corteza y Barboza et al. [7], el de la corteza junto a las hojas como remedios antidiarreicos.

A partir de los reportes de la literatura, el objetivo del presente trabajo fue aportar, mediante la experimentación científica, datos que permitan contribuir a la validación de su uso para el tratamiento de las diarreas. A tal fin, utilizamos el extracto acuoso de la corteza de J. rhombifolia, el extracto acuoso de las hojas y el conjunto de ambos extractos para ensayar los efectos resultantes en la actividad intestinal de ratones Rockland.

\section{Materiales y métodos}

\section{Material vegetal}

Las partes de la especie vegetal fueron colectadas en la provincia de San Luis, dpto. Coronel Pringles, localidad Fraga, en el establecimiento Los Chañares. La identificación botánica de la especie fue efectuada por medio de la aplicación de los métodos taxonómicos clásicos y certificada por la profesora M. E. Petenatti del Herbario de la Universidad Nacional de San Luis, Argentina (UNSL). Los ejemplares fueron colectados por triplicado, preparados y depositados en el Herbario unsL bajo el registro N.o 517.

La colección del material vegetal fue aprobada por el jefe del Programa de Biodiversidad del Ministerio de Medio Ambiente del gobierno provincial de San Luis (Resolución N. ${ }^{\circ}$ 588-PBD-2014).

\section{Preparación de los extractos}

\section{Extracto acuoso liofilizado \\ de las hojas (EALH)}

Las hojas frescas colectadas fueron minuciosamente seleccionadas, desechando todas aquellas que presentaran interrupciones en la integridad de la lámina foliar, ataques de insectos, cicatrices de golpes, etc. El remanente escogido se sometió a desecación al aire libre por un periodo de aproximadamente tres semanas, hasta peso constante. El material vegetal desecado fue molido a polvo y se preparó la infusión con agua destilada en proporción 1:10, siguiendo la metodología expuesta en la Farmacopea Nacional Argentina [30]. La infusión fue obtenida por adición del agua destilada hirviendo al material vegetal pulverizado y se dejó reposar a temperatura ambiente por un lapso de veinte minutos. El material vegetal agotado fue separado por filtración y el extracto acuoso fue concentrado y liofilizado para preservarlo.

\section{Extracto acuoso liofilizado de la corteza (EALC)}

La corteza fue minuciosamente colectada y lavada. Se desecó al aire libre hasta peso constante, se redujo a polvo fino y se preparó la decocción con agua destilada en proporción 1:10, siguiendo la 
metodología expuesta en la Farmacopea Nacional Argentina [30]. La decocción fue obtenida colocando el agua destilada y el material vegetal pulverizado en un vaso de precipitación, calentado hasta ebullición y mantenido por un lapso de veinte minutos. El material vegetal agotado por la extracción fue separado por filtración; el extracto acuoso fue concentrado y liofilizado para su preservación.

\section{Animales}

Setenta ratones Rockland de 20-30 g de peso y de ambos sexos, obtenidos del Bioterio de la Facultad de Química, Bioquímica y Farmacia de la UnsL, fueron alojados en cajas plásticas estándar con viruta de madera esterilizada y libre acceso al alimento y agua, en un ciclo día-noche de doce horas (iluminación desde la hora 07:00 a 19:00), a temperatura constante de $22 \pm 3{ }^{\circ} \mathrm{C}$ (con periódicos ciclos de cambio de aire) y a una humedad relativa de 50-60\%. Los animales fueron mantenidos bajo estas condiciones, para su aclimatación, durante tres días antes del comienzo de las experiencias.

El cuidado de los animales y los procedimientos experimentales se efectuaron en cumplimiento de la Disposición N. ${ }^{\circ}$ 6344/96 de la Administración Nacional de Medicamentos, Alimentosy Tecnología Médica (ANMAT) [31], y fueron autorizados por el Comité Institucional para el Cuidado y Uso de Animales de Laboratorio (CICUA) de la UNSL (Protocolo N.o F-89/13 en Resolución 010-14).

\section{Procedimiento experimental}

Las experiencias fueron llevadas a cabo según el método propuesto por Di Carlo et al. [32] en ratones Rockland, con modificaciones. Los animales fueron colocados en ayuno dieciocho horas antes del comienzo de las experimentaciones, con libre acceso al agua y alojados en jaulas apropiadas para evitar la coprofagia.

Los animales fueron divididos en los siguientes grupos $(n=7)$ :

- Grupo 1: control, administrado con la solución salina.

- Grupos 2, 3 y 4: experimentales, administrados con EALC a las dosis de 125, 250 y $500 \mathrm{mg} / \mathrm{kg}$ de peso.
- Grupos 5, 6 y 7: experimentales, administrados con EALH a las dosis de 125, 250 y $500 \mathrm{mg} / \mathrm{kg}$ de peso.

- Grupos 8, 9 y 10: experimentales, administrados con EALC + EALH a las dosis de 125, 250 y $500 \mathrm{mg} / \mathrm{kg}$ de peso.

En los grupos de experimentación 8, 9 y 10, la dosis calculada según el peso del animal se dividió en partes iguales de EALC y EALH para cada una de las tres dosis, procediéndose a su administración conjunta; por eso, el volumen dosificado representa la mitad de la dosis a cada extracto (por ej.: la dosis de $125 \mathrm{mg} / \mathrm{kg}$ significa $62,5 \mathrm{mg} / \mathrm{kg}$ de EALC y $62,5 \mathrm{mg} / \mathrm{kg}$ de EALH).

Los animales fueron administrados con la solución salina o extracto, según corresponda, por vía oral mediante una sonda orogástrica y a los sesenta minutos se administró una suspensión de carbón al 5\% suspendido en goma arábiga al 10\% $(0,1 \mathrm{ml} / 10 \mathrm{~g}$ de ratón). Los animales fueron sacrificados en los veinte minutos posteriores. Se removió rápidamente el intestino delgado desde el esfínter pilórico hasta la válvula ileocecal, se inspeccionó y determinó la medida de la distancia recorrida por la suspensión de carbón al 5\% desde el esfínter pilórico y hasta la posición más distal.

Para la determinación porcentual del tránsito intestinal, se utilizó la siguiente fórmula:

Distancia recorrida por la suspensión de carbón al 5\% (cm) x 100 .

Longitud total del intestino delgado $(\mathrm{cm})$.

\section{Análisis estadístico de los resultados}

Se utilizaron los programas estadísticos GraphPad Prism versión 5.00 para Windows y GraphPad InStat versión 3.00 para Windows (GraphPad Software, San Diego, California, Estados Unidos). Las figuras fueron creadas con GraphPad Prism versión 5.00 .

Se aplicó un análisis de AnOva simple y cuando fue significativo, se realizó la comparación múltiple de las medias por empleo del Tukey-Kramer Multiple Comparisons Test. Los resultados se expresaron como media \pm SEM (error estándar de la media). Una probabilidad de $p<0,05$ fue considerada significativa. 


\section{Resultados}

En el grupo de animales control $(n=7)$, la distancia atravesada por la suspensión de carbón fue 66,86 $\pm 2,50 \%$ de la longitud total del intestino delgado.

El tratamiento con EALH en los grupos experimentales 5,6 y $7(n=7)$ produjo una disminución del 13,53\%, 15,12\% y 15,82\% para las dosis ensayadas $(125,250$ y $500 \mathrm{mg} / \mathrm{kg}$, respectivamente; Figura 1). Los resultados obtenidos no fueron estadísticamente significativos versus el grupo control.

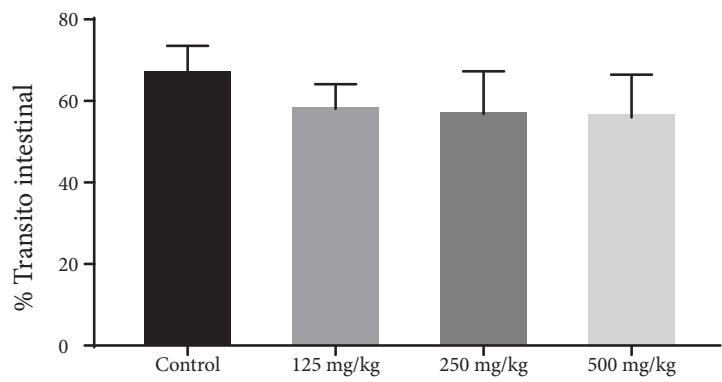

Figura 1. Efecto del tratamiento con EALH (extracto acuoso liofilizado de las hojas) de J. rhombifolia a las dosis de 125, 250 y $500 \mathrm{mg} / \mathrm{kg}$ sobre el tránsito intestinal en ratones Rockland. Los resultados representan la media \pm SEM de siete animales para cada grupo experimental.

Fuente: elaboración propia con el programa GraphPad Prism versión 5.00 con base en los resultados obtenidos

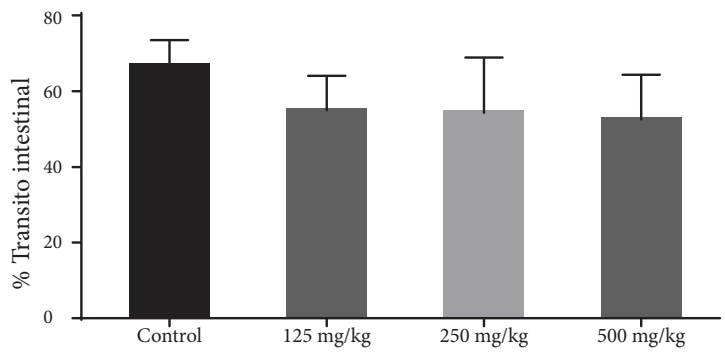

Figura 2. Efecto del tratamiento con EALC (extracto acuoso liofilizado de la corteza) de J. rhombifolia a las dosis de 125, 250 y $500 \mathrm{mg} / \mathrm{kg}$ sobre el tránsito intestinal en ratones Rockland. Los resultados representan la media \pm SEM de siete animales para cada grupo experimental.

Fuente: elaboración propia con el programa GraphPad Prism versión 5.00 con base en los resultados obtenidos

El tratamiento con EALC en los grupos experimentales 2,3 y $4(\mathrm{n}=7)$ a las dosis de 125,250 y $500 \mathrm{mg} / \mathrm{kg}$ ocasionaron una reducción del 17,81\%,
$18,69 \%$ y $21,34 \%$ del tránsito intestinal, respectivamente (Figura 2). Los resultados obtenidos no fueron estadísticamente significativos versus el grupo control.

La administración conjunta de EALC + EALH en los grupos experimentales 8,9 y $10(n=7)$ resultó en una disminución significativa, y de manera dependiente de la dosis, del tránsito intestinal en ratones Rockland. Las dosis ensayadas de 125, 250 y $500 \mathrm{mg} / \mathrm{kg}$ mostraron una reducción de la motilidad intestinal del 19,05\% ( $p<0,05), 21,21 \%(p<0,01)$ y $26,02 \%(p<0,01)$, respectivamente, en comparación con el grupo de control negativo (Figura 3).

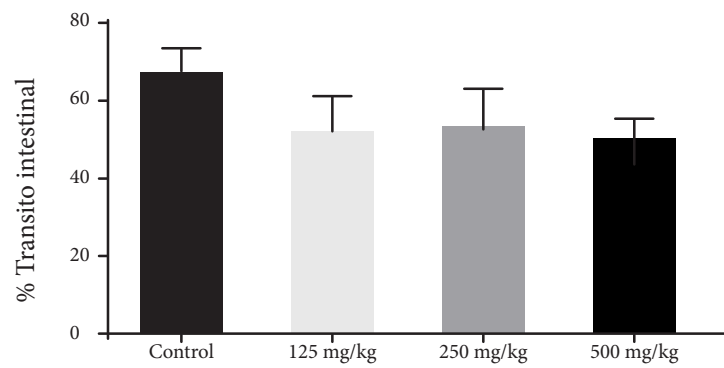

Figura 3. Efecto del tratamiento con la administración conjunta de EALC (extracto acuoso liofilizado de la corteza) + EALH (extracto acuoso liofilizado de las hojas) de J. rhombifolia a las dosis de 125, 250 y $500 \mathrm{mg} / \mathrm{kg}$ sobre el tránsito intestinal en ratones Rockland. Los resultados representan la media \pm S.E.M de siete animales para cada grupo experimental. ${ }^{\star} p<0,05 \mathrm{y}{ }^{\star *} p<0,01$ vs. control.

Fuente: elaboración propia con el programa GraphPad Prism versión 5.00 con base en los resultados obtenidos

\section{Discusión}

Los ensayos con diversos extractos vegetales activos sobre el tránsito intestinal en ratones representan un modelo de estudios farmacológicos a nivel preclínico que permite ensayar productos naturales utilizados en la medicina popular como agentes antidiarreicos, por medición de la velocidad, del tránsito intestinal normal merced a la utilización de diferentes marcadores, por ej.: una suspensión marcadora de carbón al 5\% suspendido en goma arábiga al 10\% [33].

El EALC y el EALH, por separado, no redujeron de manera significativa el tránsito intestinal normal, pero los resultados estadísticamente significativos obtenidos con la utilización conjunta de ambos 
extractos nos hacen suponer una acción sinérgica entre los constituyentes químicos contenidos en ambos órganos vegetales; y como tales encuentran consonancia con los datos manifestados por Barboza et al. [7], quienes revelan el uso conjunto de hojas y corteza para el tratamiento de la diarrea.

Además, se manifiesta el uso conjunto de hojas y corteza en la medicina popular argentina como antidisentérico [7], [22], y la justificación para el tratamiento de este trastorno inflamatorio del intestino podría recabar en su actividad reductora del tránsito intestinal normal sumada a referencia popular de la especie como antiinflamatoria en el aparato digestivo [22], [29].

La utilización con fines medicinales de las plantas propias de una región resulta una práctica común de muchas comunidades, y particularmente en la terapia antidiarreica son variadas las especies vegetales destinadas para tal fin, entre las cuales se incluye J. rhombifolia [7], [14]. Merced a ello, la investigación científica desempeña una labor de suma importancia en la validación de los usos en la medicina popular y certificando la seguridad de su utilización mediante estudios de toxicidad. Los resultados obtenidos nos permiten contribuir a la terapéutica antidiarreica confirmando que, según la metodología empleada en nuestra experimentación, el empleo conjunto de las hojas y de la corteza de J. rhombifolia provoca un efecto reductor de la motilidad intestinal.

El mecanismo de acción por el cual ambos extractos de J. rhombifolia manifiestan un efecto sinérgico reductor del tránsito intestinal normal no pueden ser explicados con los presentes datos.

Desde el punto de vista fitoquímico, las hojas contienen taninos, compuestos fenólicos, ácidos orgánicos, flavonoides, esteroides, gomas y mucílagos, y las partes aéreas contienen esteroides, triterpenoides, alcaloides, cumarinas y saponinas [7]. Por otro lado, en las hojas, Montanha et al. [34] revelaron la presencia de los $\mathrm{C}$-glicosil-flavonoides: vicenina-2, vitexina, orientina y swertisina, y Caraballo de la Peña [35] reveló la presencia de isovitexina, otra C-glicosilflavona. En cuanto a la fitoquímica de la corteza, no han sido encontrados estudios que revelen la presencia de grupos químicos particulares.

Los C-glicosil flavonoides son escasamente absorbidos en el tracto digestivo y necesitan de un tiempo prolongado de contacto con la mucosa para absorberse y ejercer su acción farmacológica [36], [37], por lo cual se desestima su participación en los resultados obtenidos en el presente estudio.

\section{Conclusión}

Los resultados obtenidos en este estudio indican que el uso conjunto del extracto acuoso de las hojas y de la corteza de J. rhombifolia posee un significante efecto reductor del tránsito intestinal normal en el modelo in vivo utilizado y que avala su utilización en la medicina popular argentina.

Por otro lado, estos resultados también nos permiten evidenciar que, mediante este modelo experimental, el extracto de las hojas y el extracto de la corteza por separado no produjeron una disminución que arroje resultados significativos con el método estadístico aplicado.

Futuros estudios deben ser llevados a cabo para la evaluación adicional de esta especie vegetal como una fuente potencial de ingredientes farmacológicamente activos en la terapia antidiarreica, que sea accesible y de bajo costo.

\section{Referencias}

[1] Organización Mundial de la Salud (oms). Estrategia de la OMs sobre medicina tradicional 2014-2023. Hong Kong: oms; 2013.

[2] Cabrera AT, Zardini EM. Manual de la flora de los alrededores de Buenos Aires. 2. edición. Buenos Aires: Acme Agency Saci y F; 1993.

[3] De la Peña MR, Pensiero JF. Plantas argentinas. Catálogo de nombres comunes. Buenos Aires: LOLA; 2004.

[4] Dimitri MJ. Enciclopedia argentina de agricultura y jardinería. 3. a edición. Buenos Aires: Acme Agency Saci y F; 2004.

[5] Alonso J, Desmarchelier CJ. Plantas medicinales autóctonas de la Argentina. Bases científicas para su aplicación en atención primaria de la salud. Buenos Aires: Fitociencia; 2015.

[6] Arias Toledo B, Galetto L, Colantonio S. Ethnobotanical knowledge in rural communities of Cordoba (Argentina): The importance of cultural and biogeographical factors. J Ethnobiol Ethnomed. 2009;5:40. doi: https://doi.org/10.1186/1746-42695-40. 
[7] Barboza GE, Cantero JJ, Núñez C, Pacciaroni A, Ariza Espinar L. Medicinal plants: A general review and a phytochemical and ethnopharmacological screening of the native Argentine flora. Kurtziana. 2009;34(1-2):7-365.

[8] Carosio MC, Junqueras MJ, Andersen A, Abad SM. Árboles y arbustos nativos de la provincia de San Luis. Buenos Aires: San Luis Libro; 2008.

[9] Carrizo EV, Palacio MO, Roic LD. Uso medicinal de algunas especies nativas en Santiago del Estero (República Argentina). Dominguezia. 2005;21(1):25-32.

[10] Filipov A. Medicinal plants of the Pilagá of Central Chaco. J Ethnopharmacol. 1994;44(3):181-93. doi: 10.1016/0378-8741(94)01185-0.

[11] Furlán V, Torres C, Galetto L. Conocimiento y utilización de plantas medicinales por pobladores rurales del bosque chaqueño serrano de Córdoba (Argentina). Bonplandia. 2011;20(2):285-307.

[12] Goleniowski ME, Bongiovanni GA, Palacio L, Núñez CO, Cantero JJ. Medicinal plants from the "Sierra de Comechingones”, Argentina. J Ethnopharmacol. 2006;107(3):324-41. doi: 10.1016/j.jep.2006.07.026.

[13] Hieronymus J. Plantae diaphoricae. Florae argentina. Buenos Aires: Editorial Guillermo Kraft; 1882.

[14] Hurrell JA, Ulibarri EA, Arenas PM, Pochettino ML. Plantas de herboristería: plantas medicinales que se comercializan en herboristerías de la ciudad de Buenos Aires. Buenos Aires: LOLA; 2011.

[15] Lahitte HB, Hurrell JA. Plantas medicinales rioplatenses. Buenos Aires: LolA; 1998.

[16] Madaleno IM. Organic cultivation and use of medicinal plants in Latin America. Pharmacognosy Communications. 2012;2(4):34-51. doi: https://doi. org/10.5530/pc.2012.4.7.

[17] Martínez GJ. Medicinal plants used by the Criollos of Calamuchita to treat blood, cardiovascular, and neuroendocrinous diseases. J Herbs Spices Med Plants. 2007;13(3):55-82. doi: https:// doi.org/10.1300/j044v13n03_06.

[18] Martínez GJ. Uso de plantas medicinales en el tratamiento de afecciones transmitidas por el agua en una comunidad toba (Qom) del Impenetrable (Chaco, Argentina): una perspectiva etnoecológica y sanitaria. Bonplandia. 2011;20(2):329-52.

[19] Martínez Crovetto R. Las plantas utilizadas en medicina popular en el no de Corrientes (República Argentina). Miscelánea Fundación Miguel Lillo. 1981;69:1-139.

[20] Menseguez P, Galetto L, Anton AM. El uso de plantas medicinales en la población campesina de El Puesto (Córdoba, Argentina). Kurtziana. 2007;33(1):89-102.
[21] Núñez C, Cantero JJ. Las plantas medicinales del sur de la provincia de Córdoba. Río Cuarto: Editorial de la Fundación Universidad Nacional de Río Cuarto; 2000.

[22] Ratera EL, Ratera MO. Plantas de la flora argentina empleadas en medicina popular. Buenos Aires: Hemisferio Sur; 1980.

[23] Roig FA. Flora medicinal mendocina. Las plantas medicinales y aromáticas de la provincia de Mendoza (Argentina). Mendoza: EdIUnC; 2002.

[24] Sharry S, Abedini W, Basiglio Cordal MA, Briones V, Roussy L, Stevani R, et al. Food and medicinal value of some forest species from Buenos Aires (Argentina). Emirates Journal of Food and Agriculture. 2011;23(3):222-36.

[25] Toursarkissian M. Plantas medicinales de la Argentina. Sus nombres botánicos, vulgares, usos y distribución geográfica. Buenos Aires: Hemisferio Sur; 1980.

[26] Vischi N, Arana M. Utilidad de las plantas autóctonas del Espinal. Río Cuarto: Fundación Universidad Nacional de Río Cuarto; 2002.

[27] Verettoni HN. Contribución al conocimiento de las plantas medicinales de la región de Bahía Blanca. Bahía Blanca: Universidad Nacional del Sur; 1985.

[28] Giménez AM, Moglia JG, Hernández P, Gerez R. La factibilidad de incrementar el valor de los bosques del Chaco mediante el aprovechamiento de la corteza forestal. Quebracho Revista de Ciencias Forestales. 2008;15:9-14.

[29] Demaio P, Karlin UO, Medina M. Árboles nativos de Argentina. Tomo 1: Centro y Cuyo. Córdoba: Ecoval; 2015.

[30] Farmacopea Nacional Argentina. Codex medicamentarius argentino. 6. ${ }^{a}$ edición. Buenos Aires: Farmacopea Nacional Argentina; 1978.

[31] Administración Nacional de Medicamentos, Alimentos y Tecnología Médica (ANMAT). Disposición N. ${ }^{\circ}$ 6344/96. Buenos Aires, Argentina. Boletín Oficial de la Nación N. ${ }^{\circ}$ 28567, Primera Sección (20 ene 1997).

[32] Di Carlo G, Mascolo N, Izzo A, Capasso F, Autore G. Effects of quercetin on the gastrointestinal tract in rats and mice. Phytotherapy Research. 1994;8:42-5. doi: https://doi.org/10.1002/ptr.2650080110.

[33] Nunes Salgado HR. Sección generalidades: capacitación en metodologías biofarmacéuticas. En González Álvarez I, Cabrera Pérez MA, Bermejo Sanz M. Metodologías biofarmacéuticas en el desarrollo de medicamentos. Buenos Aires: Universitas Miguel Hernández; 2015. 
[34] Montanha JA, Schenkel EP, Cardoso-Taketa AT, Dresch AP, Langeloh A, Dallegrave E. Chemical and anti-ulcer evaluation of Jodina rhombifolia (Hook. \& Arn.) Reissek extract. Brazilian Journal of Pharmacognosy. 2009;19(1A):29-32. doi: https:// doi.org/10.1590/s0102-695x2009000100007.

[35] Caraballo de la Peña D. Cultivo de callos in vitro a partir de las hojas de Jodina rhombifolia Hook. \& Arn. (Reissek) para obtención de C-glicosilflavonas [tesis doctoral]. [La Plata]: Universidad Nacional de la Plata, Argentina; 2015.
[36] Talhi O, Silva AMS. Advances in C-glycosylflavonoid research. Current Organic Chemistry. 2012; 16(7):859-96. doi: https://doi.org/10.2174/1385272 12800194791.

[37] Zeng P, Zhang Y, Pan C, Jia Q, Guo F, Li Y, et al. Advances in studying of the pharmacological activities and structure-activity relationships of natural C-glycosylflavonoids. Acta Pharmaceutica Sinica B. 2013;3(3):154-62. doi: https://doi.org/10.1016/j. apsb.2013.04.004. 